\title{
Projeto de controle para um sistema Viga-Bola
}

\author{
Mariana Aparecida de Moraes*. Jean Marcos de Souza Ribeiro*. \\ Carlos Antônio Alves*.
}

\author{
*UNESP - Universidade Estadual Paulista, Campus de Ilha Solteira \\ Departamento de Engenharia Elétrica, Faculdade de Engenharia de Ilha Solteira. \\ Ilha Solteira-SP, Brasil, (e-mails: mariy_ap@yahoo.com.br, jean.ribeiro@unesp.br, carlos.a.alves@unesp.br).
}

\begin{abstract}
This work consists in the construction of a platform that cooperates for the learning of classic control concepts, using for this a naturally unstable and nonlinear plant. The Ball \& Beam system was built with a servo motor, a beam engaged on the servo motor shaft and an optical sensor at the end of the beam. The servo works the angular positioning of a beam, controlling the desired position of a sphere with the aid of a distance sensor, and a microcontroller for the control implementation. A supervisory software for signal acquisition and bench results analysis was used. The result of this work has a strong didactic appeal and can be used for undergraduate and postgraduate education.
\end{abstract}

Resumo: Este trabalho consiste na construção de uma plataforma que coopera para o aprendizado de conceitos de controle clássico, utilizando para isso uma planta naturalmente instável e não linear. O sistema Viga-Bola foi construído com um servo motor, uma viga engastada no eixo do servo motor e um sensor ótico na extremidade da viga. O servo trabalha o posicionamento angular de uma viga, controlando a posição desejada de uma esfera, com o auxílio de um sensor de distância, e um microcontrolador para a implantação do controle. Foi utilizado um software supervisório para aquisição de sinais e análise de resultados de bancada. $\mathrm{O}$ resultado deste trabalho tem forte apelo didático e poderá ser aproveitado para o ensino de graduação e pós-graduação.

Keywords: Arduino. Ball and Beam System. PID. Control.

Palavras-chaves: Arduino. Sistema Viga-Bola. PID. Controle.

\section{INTRODUÇÃO}

O controle de sistemas tem muita importância no avanço da engenharia, dado sua relevância na robótica, instrumentação, assim como no controle automatizado das plantas industriais. Controlar um sistema significa agir sobre ele de modo a buscar um resultado desejado.

Uma vez que a natureza é dinâmica, grande parte dos sistemas são não lineares. Porém, pode-se realizar uma linearização do sistema aproximando-os por modelos matemáticos que circundam um ponto de equilíbrio e normalmente limitam-se em faixas de atuação.

Através da linearização é possível provar a existência de estabilidade em alguns sistemas não lineares em uma região em torno de um ponto de operação. Assim, pode-se projetar um controlador para um sistema não linear utilizando a teoria de controle clássica (OGATA, 2010).

\subsection{Sistemas Viga-Bola (Ball \& Beam)}

Em um sistema Viga-Bola a posição da bola na viga é controlada pelo servo motor que fornece o movimento de rotação à viga. A bola se move de acordo com o ângulo gerado pelo movimento do servo. O controle desta planta tem atraído a atenção de vários pesquisadores por ser um sistema instável, por apresentar não linearidades e incertezas, como descritos em Yi et al. (2001), Turker et al. (2007), Diez et al. (2009), Rana et al. (2011), Jain \& Nigam (2013), Anjali \& Mathew (2016) e Oliveira et al. (2017).

Em Turker et al. (2007) é apresentado um trabalho que utiliza o controle adaptativo direto e robusto com incertezas paramétricas. Já em Diez et al. (2009) os autores sugerem a implementação de um laboratório virtual e remoto utilizando o sistema viga-bola e a técnica de controle PID. Em Diez et al. (2009) é descrito a utilização do sistema Viga-Bola para a conceituação e desenvolvimento matemático da modelagem do sistema Ball-Balancer. Ainda, em Yi et al. (2001), é proposto um novo controlador fuzzy para a estabilização de sistemas Ball \& Beam baseado em SIRM (single-input ruler module) dinamicamente conectado ao modelo de inferência fuzzy. Em 2017 é proposto, em Oliveira et al. (2017), uma forma de obter a posição da bola utilizando um sensor de corrente, em que esta, sendo metálica, faz um contato elétrico através de dois trilhos $\mathrm{Cr}-\mathrm{Ni}$ nos quais ela circula.

Em Anjali \& Mathew (2016) utiliza-se a estratégia de controle clássica PID para controle da posição da esfera e posição angular da viga com o uso de um servo motor, em que os parâmetros de controle são ajustados por meio de um Algoritmo Genético. Por fim, em Rana et al. (2011), é apresentada uma técnica de otimização robusta para ajustar os ganhos do controlador PID através do algoritmo Particle 
Swarm Optimization (Otimização do Enxame de Partículas) que emula o comportamento do bando de aves.

A linearização em torno do ponto de operação pode ser aplicada ao sistema, porém deve-se atentar que esta pode ser apenas uma solução local. No entanto, é muito boa para aplicações em regiões de pequenas atrações, mas pode falhar em uma escala maior (TURKER et al., 2007).

Neste trabalho foi realizado a construção de uma planta, a modelagem matemática dela, o projeto de um controlador clássico PID, com simulações através do software Matlab/ Simulink e implementação em bancada do controle através de utilização de uma plataforma de prototipagem Arduino UNO na etapa de controle e um sensor infravermelho para a verificação da posição da esfera. Os resultados demonstram a possibilidade de utilização do sistema, com finalidade de estudos em nível de graduação e pós-graduação.

\subsection{Modelo Linear da Planta Viga-Bola (Ball \& Beam)}

Na Fig. 1 é ilustrada a composição básica do sistema VigaBola construído neste trabalho. No sistema ilustrado, a bola geralmente está a uma distância $x(t)$ metros do pivô central, mas para este trabalho considera-se o zero no ponto onde se localiza o sensor de distância, e a viga é rotacionada a um ângulo $\theta$, medido em radianos.

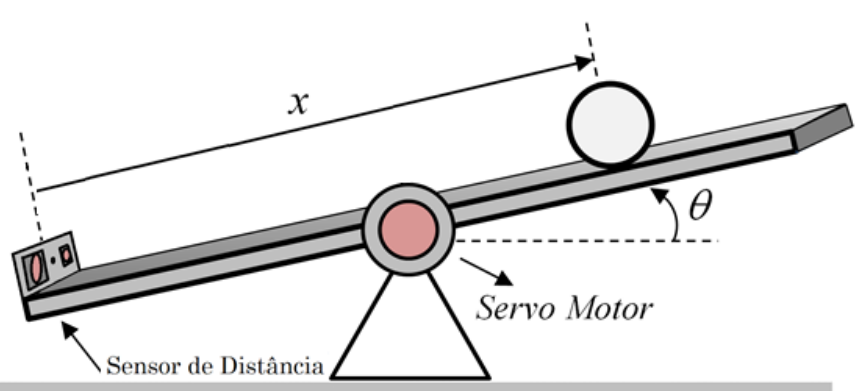

Fig. 1. Desenho esquemático de um sistema Ball \& Beam.

O sistema em espaço de estados, que representa este modelo, é descrito em (1) e (2).

$$
\begin{aligned}
& x \dot{(t)}=\left[\begin{array}{cccc}
0 & 1 & 0 & 0 \\
0 & 0 & 3 g / 7 & 0 \\
0 & 0 & 0 & 1 \\
0 & 0 & 0 & -\frac{1}{\tau}
\end{array}\right]\left[\begin{array}{c}
x(t) \\
\dot{x}(t) \\
\theta(t) \\
\dot{\theta}(t)
\end{array}\right]+\left[\begin{array}{c}
0 \\
0 \\
0 \\
\frac{K_{\text {motor }}}{\tau}
\end{array}\right] u(t), \\
& y(t)=\left[\begin{array}{llll}
1 & 0 & 0 & 0
\end{array}\right]\left[\begin{array}{c}
x(t) \\
\dot{x}(t) \\
\theta(t) \\
\dot{\theta}(t)
\end{array}\right]
\end{aligned}
$$

sendo $\mathrm{g}$ a constante gravitacional, $\tau$ a constante de tempo do motor e $K_{\text {motor }}$ a constante relacionada à fabricação do mesmo dados em Quanser, 2008.

\section{MÉTODO DE CONTROLE}

Nesta seção serão apresentados os métodos de controle PID e de Alocação de polos.

\subsection{Controlador PID}

A utilidade dos controles PID está na sua aplicabilidade geral à maioria dos sistemas de controle. Em particular, quando o modelo matemático da planta não é conhecido e, portanto, métodos de projeto analíticos não podem ser utilizados, controles PID se mostram os mais úteis, devido a regras de sintonia, que permitem fazer o ajuste fino do controlador (OGATA, 2010). O controlador para a posição da bola é do tipo PID e seu diagrama de blocos é ilustrado na Fig. 2.

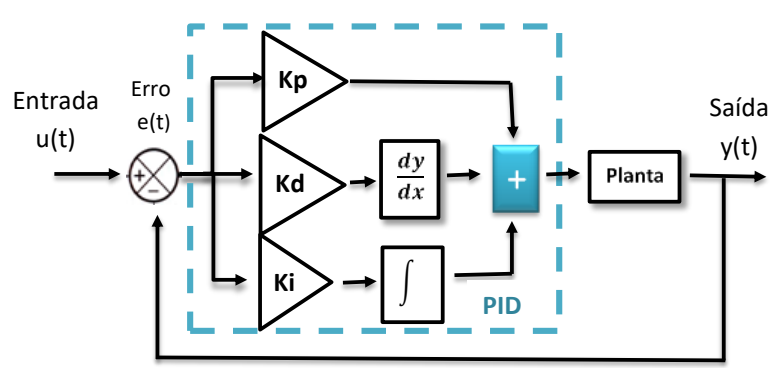

Fig. 2. Controle PID de uma planta.

A função de transferência do bloco "PID" e sua equação de saída no domínio do tempo são representadas por (3) e (4), respectivamente:

$$
\begin{gathered}
G_{c}(s)=K_{P}+\frac{K_{I}}{s}+K_{D} s \\
u(t)=K_{P} e(t)+K_{I} \int e(t) d t+K_{D} \frac{d e(t)}{d t} .
\end{gathered}
$$

O controlador PID apresenta bom desempenho em uma ampla faixa de condições de operação e simplicidade em sua funcionalidade. Para realizar sua implementação é necessário determinar os ganhos para um determinado processo. Ainda tem em sua característica um polo na origem e dois zeros $\mathrm{z}_{1} \mathrm{e}$ $\mathrm{z}_{2}$ que podem ser posicionados em qualquer lugar do semiplano s esquerdo (DORF \& BISHOP, 2011).

$$
\begin{aligned}
& G c(s)=K_{1}+\frac{K_{2}}{s}+K_{3} s=\frac{K_{3} s^{2}+K_{1} s+K_{2}}{s}=\frac{K_{3}\left(s^{2}+a s+b\right)}{s}= \\
& \frac{K_{3}\left(s+z_{1}\right)\left(s+z_{2}\right)}{s}
\end{aligned}
$$$$
\text { sendo } a=\frac{K_{1}}{K_{3}} \text { e } b=\frac{K_{2}}{K_{3}} \text {. }
$$

\section{DESENVOLVIMENTO DO PROTÓTIPO}

A viga do sistema tem acoplamento com o servo motor por meio de duas engrenagens. O sensor infravermelho está acoplado em uma das extremidades da viga. Há um obstáculo em $10 \mathrm{~cm}$, após a localização do sensor, constituído de uma espuma e fita isolante para que a esfera não ultrapassasse distâncias menores que $10 \mathrm{~cm}$ do sensor, uma vez que estas leituras apresentam erro de precisão conforme descrito no datasheet do sensor de ótico (SHARP CORPORATION, 2006).

O protótipo finalizado está ilustrado na Fig. 3. 


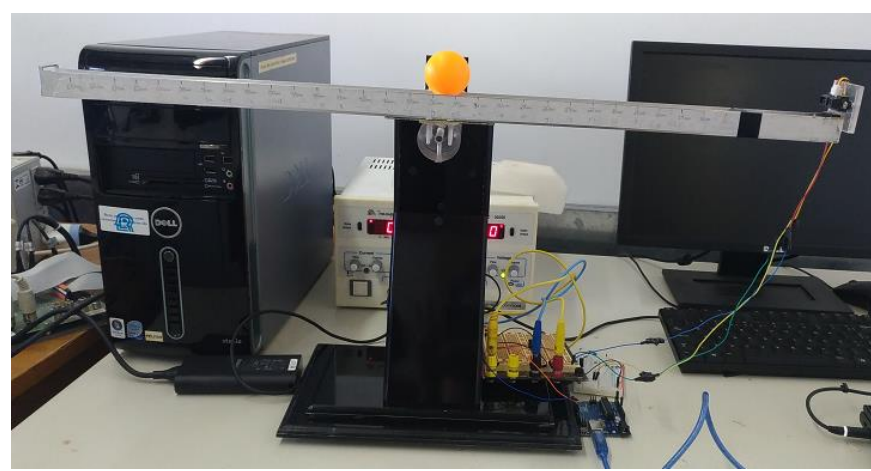

Fig. 3. Protótipo Viga-Bola desenvolvido em laboratório

\subsection{Implementação do controlador PID no microcontrolador}

Tem-se que o sinal de controle gerado pelo PID se dá por (6) e que o erro $e(t)$ é a diferença entre a posição desejada (setpoint) e a posição atual da esfera, dada por (7).

$$
\begin{gathered}
u(t)=K_{p} e(t)+K_{i} \int e(t) d t+K_{d} \frac{d e(t)}{d t}, \\
\text { erro: } e(t)=\text { setpoint }-x(t) .
\end{gathered}
$$

Dessa forma, primeiramente é calculado o erro $e(t)$ com o auxílio do sensor infravermelho e então calcula-se a derivada e a integral do mesmo, multiplicando-os individualmente pelos seus ganhos. A equação (8) representa o cálculo realizado para obtenção da contribuição derivativa.

$$
\frac{d \text { Erro }}{d t}=\frac{d \text { setpoint }}{d t}-\frac{d x(t)}{d t} .
$$

Entretanto, uma vez que o valor de setpoint é constante, temos que sua derivada é igual a zero. Dessa forma, tem-se que:

$$
\frac{d E r r o}{d t}=-\frac{d x(t)}{d t}
$$

Dessa maneira, a equação (9) apresenta uma solução para a implementação prática no microcontrolador. No ambiente digital, não é trabalhado com um tempo contínuo, para isso $\Delta t=1 / f_{\text {amostragem }}$ isto é, $\Delta t$ é o tempo de amostragem. Dessa forma, utilizou-se o controle PID no microcontrolador, começando pelo termo proporcional de (6). Multiplica-se o termo proporcional $K p$ pela variável erro no instante $t$. A implementação é feita no domínio de tempo discreto e é dada por (10).

$$
K p . e(t) \leftrightarrow K p . e[n] .
$$

A contribuição derivativa do PID na equação (6), no domínio de tempo discreto, segue em (11) baseando em (9) e adicionando-se o ganho $K d$ :

$$
K d . \frac{d e(t)}{d t} \leftrightarrow K d .(-e[n-1])
$$

O termo integral segue a lógica inversa do termo derivativo; agora existirá uma somatória com intervalos de tempo finitos expressa em (12).
$\int_{t}^{t+\Delta t} f(t) d t=\lim _{\Delta \vec{t} 0} \sum_{n=t}^{t+\Delta t} f(n) \Delta t=\Delta t .\{f(t)+f(t+$ $\Delta t)\} .(12)$

Ainda utilizando $\Delta \mathrm{t}$ como o tempo de amostragem, e sendo suficientemente pequeno, tem-se (13).

$$
\int_{n}^{n+\Delta \mathrm{n}} f[n] d n=f[n]+f[n-1]
$$

O termo integral de (6) é dada por (14).

$$
K i \int_{t=0}^{t} e(t) d t=K i \cdot\{e[n]+e[n-1]\}
$$

A equação final discretizada é representada por (15).

1]),

$$
u[n]=K p \cdot e[n]+K d \cdot(-e[n-1])+K i \cdot(e[n]+e[n-
$$

sendo $K i=\frac{K i \cdot t_{\text {amostragem }}}{t_{i}}$ e $K d=\frac{K d \cdot t_{d}}{t_{\text {amostragem }}}$.

\subsection{Sensor Infravermelho}

O dispositivo para medição de distância utilizado neste projeto é um sensor infravermelho do fabricante SHARP, modelo GP2Y0A21YK0F. De acordo com seu manual, tem-se a seguinte característica de saída, dada pela Fig. 4.

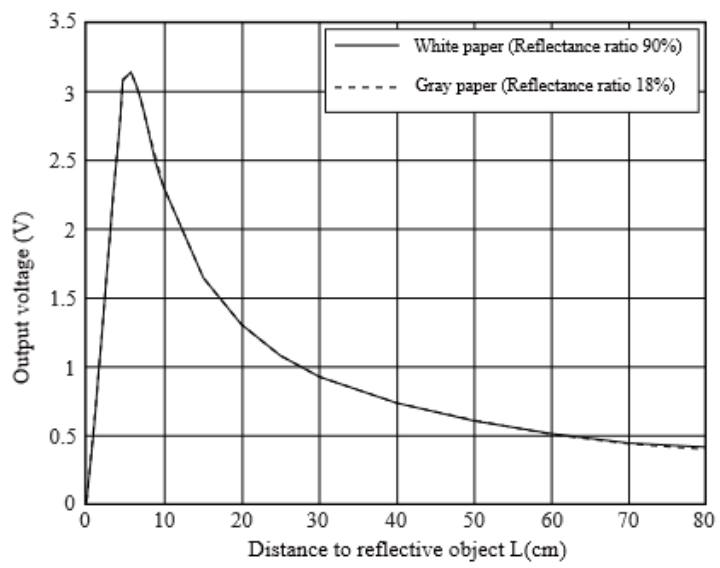

Fig. 4. Curva característica do sensor (SHARP CORPORATION, 2006).

Este dispositivo produz tensão correspondente à distância detectada e possui um alcance de medição de 10 à $80 \mathrm{~cm}$, da qual atende às necessidades do projeto uma vez que a viga possui $70 \mathrm{~cm}$. Dessa forma, foi feita uma calibração do sensor para obter as medidas no programa: verificou-se a esfera em 10 pontos da viga sendo obtido os valores pelo microcontrolador utilizando o conversor ADC de 10 bits integrado ao Arduino, montando-se o gráfico da Fig. 5. Assim, na programação faz-se a leitura da saída do sensor, em ADC, e este retorna o valor em centímetros correspondente à curva de calibração de acordo com o trecho de programação abaixo:

"int dcal $]=\{66,58,50,42,38,34,26,22,16,10\}$;

int ADCcal []$=\{184,193,197,204,213,224,262,293,372,638\}$;

For (int $i=0 ; i<9 ; i++$ ) //Curva de calibração ADC para cm.

$\{$ if (medida $>=A D C c a l[i] \& \&$ medir $<$ ADCcal $[i+1])$ 
$\{$ distcm $=$ map (medida, ADCcal $[i], A D C c a l[i+1]$, dcal $[i], d c a l[i+$ $1]) ;\}\} "$.

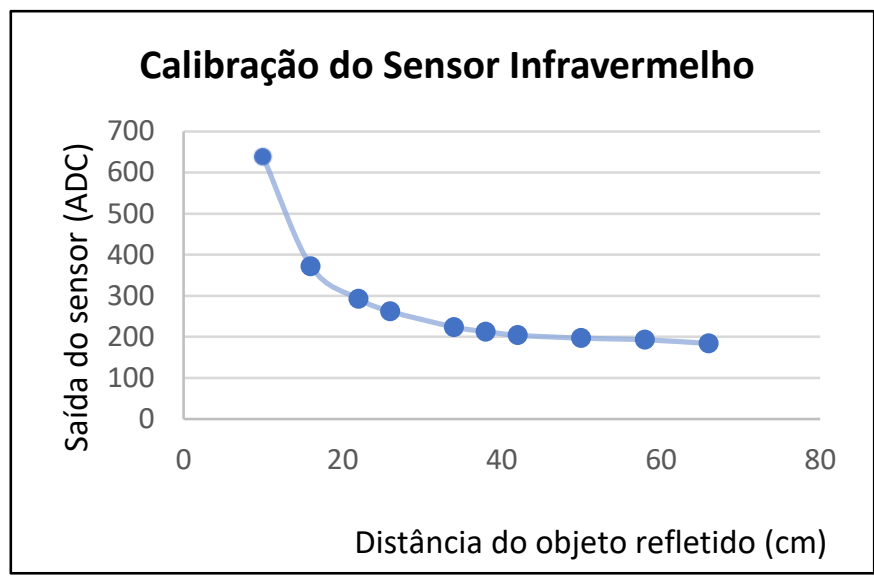

Fig. 5. Gráfico experimental da calibração do sensor infravermelho

Para melhor estabilidade da linha de suprimento, foi colocado um capacitor de $100(\mu \mathrm{F})$ entre Vcc (5V) e GND, assim como para melhor estabilidade de leitura foi inserido um capacitor de $10(\mu \mathrm{F})$ entre o sinal de saída do sensor com o GND. Observa-se, na Fig. 6, o resultado com a inserção do capacitor.

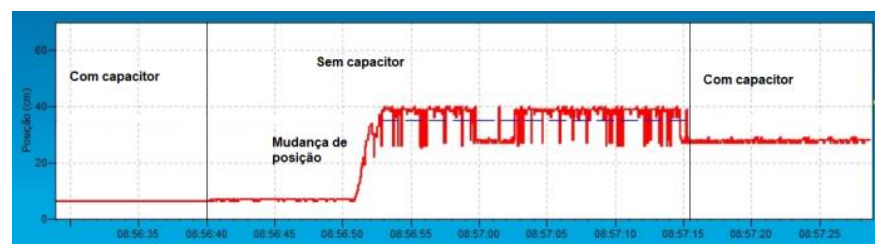

Fig. 6. Ensaios do sensor de distância com capacitor e sem.

Há uma grande mudança na qualidade e precisão das medidas, principalmente mais distantes do sensor, com o uso do capacitor, em que $10 \mu \mathrm{F}$ mostrou-se suficiente sem prejudicar notoriamente a velocidade de leitura do sensor. Os pontos adquiridos para a calibração da Fig. 5 já contava com o uso do capacitor.

\section{RESULTADOS TEÓRICOS}

Na prática não é possível ter acesso ao controle da malha interna do servo, pois já existe um controle sendo realizado quando se deseja certa posição angular. Utilizou-se as especificações dadas por Riva (2015) conforme informações a seguir:

- P.O.BB $=5 \%$ - [Porcentagem de overshoot da viga $]$.

- T. estabilização: 1,5 s - [Tempo de estabilização].

- M.E.T. estabilização: 7\% - [Margem de erro do tempo de estabilização].

- $\quad$ T.p. $=1 \mathrm{~s}-$ [Tempo de decaimento do polo Po].

- $\quad$ T.r. $=0,001 \mathrm{~s}-[$ Tempo de reset do sistema antiwindup]. INT.

- $\mathrm{MAX}=1,6$ - [Saturação máxima do integrador].

Os valores encontrados dos ganhos são: $K_{p}=2,08(\mathrm{rad} / \mathrm{m}), K_{i}$ $=1,41(\mathrm{rad} / \mathrm{m}), K_{d}=0,84(\mathrm{rad} / \mathrm{m})$. Convertendo os ganhos para valores compatíveis com as grandezas do projeto serão utilizadas os seguintes valores para as constantes $K_{p}=1,19$ graus/(cm.s), $K_{i}=0,80$ graus $/(\mathrm{cm} . \mathrm{s}), K_{d}=0,48$ graus $/(\mathrm{cm} . \mathrm{s})$.

Na Fig. 7 é ilustrado os resultados da simulação.
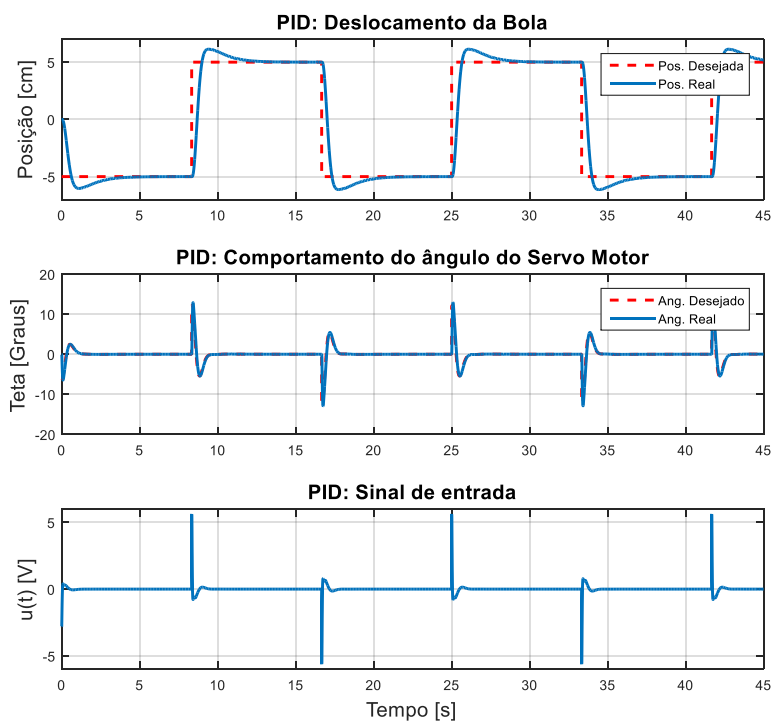

Fig. 7. Controlador PID - Resultado da simulação.

É possível observar que houve uma rápida resposta em busca da referência, porém houve um overshoot de aproximadamente $1 \mathrm{~cm}$.

Com o resultado da simulação é possível observar que o sistema apresentou estabilidade com uma resposta de aproximadamente 2 segundos.

\section{ELIPSE E3 STUDIO - RESULTADOS PRÁTICOS}

Para realizar o desenvolvimento da interface gráfica foi utilizado o software Elipse E3 Studio, possibilitando a comunicação serial com o microcontrolador em tempo real. A interface permite ao usuário observar e manipular parâmetros do sistema, como por exemplo, os ganhos do controlador PID da malha externa. Também é possível realizar o ajuste do valor de setpoint com três funções: Manual, Senoidal e Degrau.

A função Manual permite que o usuário insira o setpoint desejado manualmente, possível apenas para valores entre 10 e $69 \mathrm{~cm}$. O modo Senoidal transforma o setpoint em uma senoide, das quais período e amplitude podem ser manipulados pelo usuário. Por fim, o modo Degrau faz com que o setpoint modifique-se para um sinal degrau positivo e

negativo, periodicamente, e assim como no modo Senoidal, seus parâmetros são manipuláveis pelo usuário.

\subsection{Implementação do controlador PID}

No primeiro teste realizado, utilizou-se um controlador PID com os $K p=1$ graus $/ \mathrm{cm}, K i=50$ graus $/(\mathrm{cm} . \mathrm{s})$, e $K d=2$ graus/(cm.s). A Fig. 8 ilustra os resultados obtidos com o controlador PID com estes ganhos no modo Manual, com setpoint em $25(\mathrm{~cm})$. A posição final da esfera foi satisfatória 
com o erro nulo, porém as dificuldades físicas da planta seguraram o erro de $-1(\mathrm{~cm})$ por um longo período, ainda que o ganho integral estivesse muito alto.

Posteriormente alterou-se o setpoint para o modo senoidal com uma amplitude de $10(\mathrm{~cm})$ e um período de $30(\mathrm{~s})$. O ganho $K i$ diminuiu para 10 e $K d$ aumentou para 3, uma vez que o termo derivativo opera sobre a velocidade da bola impedindo que ela ultrapassasse muito o valor do setpoint para mais ou para menos. A Fig. 9 representa este ensaio no apêndice A.

Por fim, a Fig. 10 contém o ensaio realizado com o setpoint no modo degrau com amplitude de $12(\mathrm{~cm})$ e um período de 20 (s) em que a estabilização ocorreu em aproximadamente 6 (s).

O principal problema encontrado foi em relação ao sensor infravermelho, devido à ruídos no sinal de leitura próximas à extremidade oposta da viga. Como pode ser visto nos resultados práticos, houve alguns picos no sinal de leitura da posição da bola; estes picos atrapalham no cálculo do erro afetando diretamente o controle da viga e sendo prejudicial, principalmente, para a parte de controle derivativa.

Um limitante do servo motor era a sua incapacidade de informar sua angulação, fazendo com que o estado $\theta(t)$ e $\dot{\theta}(t)$ se tornasse desconhecidos inviabilizando a implantação da alocação de polos no trabalho, por exemplo.

\section{CONCLUSÃO}

O trabalho contemplou a modelagem matemática, linearização do modelo, simulação e implementação prática de um sistema de controle para a planta Viga-Bola.

As simulações feitas no ambiente Matlab/Simulink foram ideais, sem adição de distúrbios no sistema ou qualquer tipo de ruído; assim os resultados obtidos no controle do servo, utilizado na simulação, diferem do real utilizado no projeto.

O projeto desde o início teve como base o desenvolvimento de um protótipo com capacidade de controlar um sistema vigabola. Em geral a maioria dos componentes utilizados no projeto são de baixo custo.

Ainda que o sensor infravermelho tenha sido testado, obtendo poucos picos com o capacitor, durante os ensaios os picos persistiam de forma que a luz ambiente modificava as leituras e o sensor deveria ser calibrado sempre que essa se mostrava mais ou menos intensa.

Dessa forma, o trabalho, ainda que com resultados satisfatórios, pode ser aprimorado com a melhora do sistema físico para redução dos distúrbios do sensor de distância e um motor CC com encoder, por exemplo, para que fosse possível a obtenção do ângulo $\theta$ e sua função transferência. Analisando os resultados práticos é possível observar que o controlador PID manteve a bola em torno do setpoint, comprovando assim a possibilidade do desenvolvimento de um protótipo de controle do sistema viga-bola utilizando componentes de baixo custo. Para trabalhos futuros, sugere-se a utilização de um controlador por Alocação de polos e Fórmula de Ackermann utilizando um giroscópio ou um acelerômetro para se obter os estados. Assim, o trabalho se apresenta útil para fins didáticos com facilidade de reprodução da planta e de seus controles.

\section{REFERÊNCIAS}

ALVES, U. N. L. T. (2014). Aplicação de Controle com Modos Deslizantes em Sistemas com Atraso na Aquisição de Dados. Dissertação de Mestrado. Faculdade de Engenharia de Ilha Solteira (FEIS / UNESP). 125p, Ilha Solteira-SP.

ANJALI, T; MATHEW, S. S. (2016) Implementation of optimal control for ball and beam system. International Conference on Emerging Technological Trends (ICETT).

DIEZ, J. L.; VALLES, M.; VALERA, A. (2009). Virtual and Remote Laboratory of the Ball and Beam System.

J. OLIVEIRA; S. BALULA; H. FERNANDES. (2017). Ball \& beam experiment control with current sensing. 4th Experiment@International Conference.

J. YI; N. YUBAZAKI ; K. HIROTA. (2001). Stabilization control of ball and beam systems. 9th IFSA World Congress and 20th NAFIPS International Conference.

JAIN, P.; NIGAM, M.J. (2013). Real Time Control of Ball and Beam System with Model Reference Adaptive Control Strategy using MIT Rule. IEEE International Conference on Computational Intelligence and Computing Research.

OGATA, K. (2010). Engenharia de Controle Moderno. Person, 4 a ed. São Paulo.

QUANSER. (2008). 2D Ball Balancer Control using QUARC - Instructor Manual. Canada: 87 p.

RANA, M. A; USMAN, Z; SHAREEF, Z. (2011). Automatic control of ball and beam system using Particle Swarm Optimization. IEEE 12th International Symposium on Computational Intelligence and Informatics (CINTI).

RIVA, M. F. (2015). Desenvolvimento de protótipo e controle de sistema viga-bola. Trabalho de Graduação. Faculdade de Engenharia de Ilha Solteira (FEIS / UNESP). Unpublished.

SHARP CORPORATION (2006). Datasheet: SHARP GP2Y0A21YK0F.

TURKER, T.; GORGUN, H.; ZERGEROGLU, E.; CANSEVER, G. (2007). Exact Model Knowledge and Direct Adaptive Controllers on Ball and Beam. Proceedings of International Conference on Mechatronics Kumamoto Japan.. 
Apêndice A. Resultados Práticos

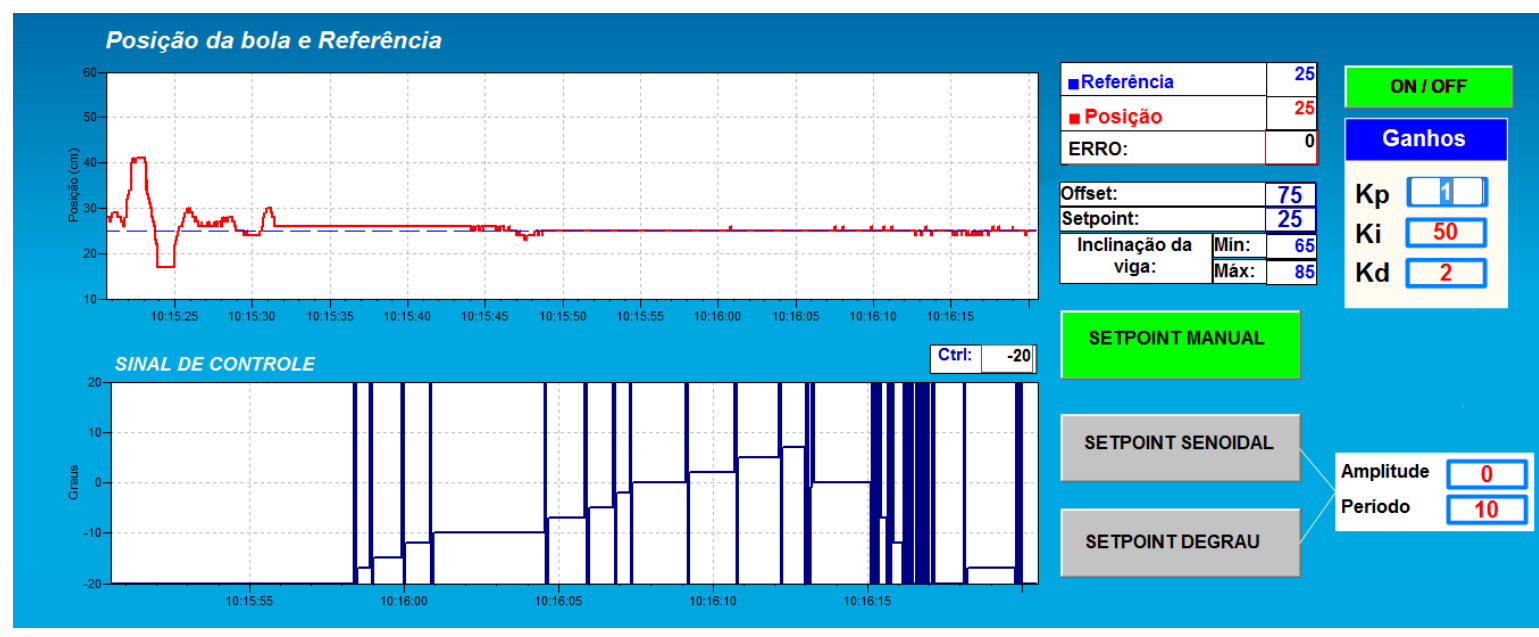

Fig. 8 Controlador PID - Setpoint Manual.

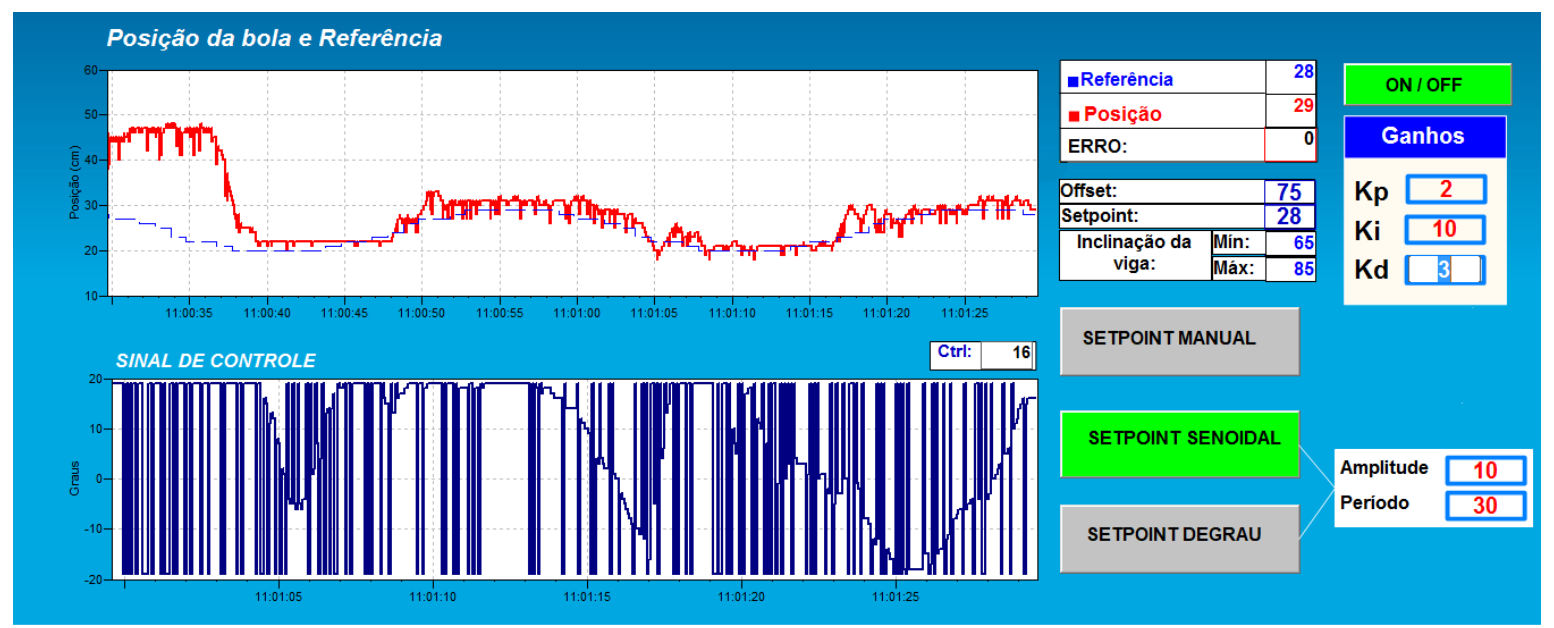

Fig. 9 Controlador PID - Setpoint senoidal com período de 30 segundos.

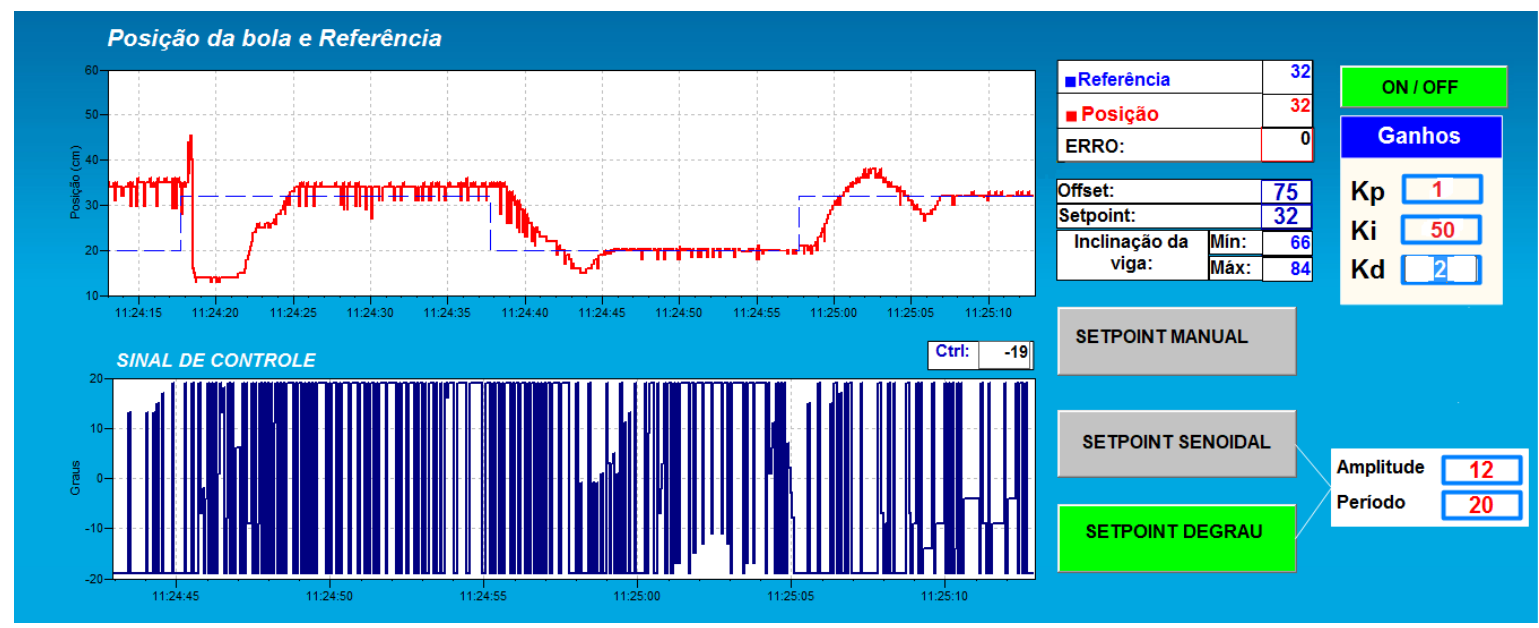

Fig. 10 Controlador PID - Setpoint degrau. 